Note

\title{
Changes in Urinary Excretions of C-Telopeptide and Cross-Linked N-Telopeptide of Type I Collagen during Pregnancy and Puerperium
}

\author{
AKIHIRo YAMAGA, MichiYoshi TAGA, AND Hiroshi MINAGUCHI \\ Department of Obstetrics and Gynecology, Yokohama City University School of Medicine, Yokohama 236, Japan
}

\begin{abstract}
Previously we reported an increase in bone resorption during pregnancy and lactation by measuring pyridinoline (Pyr) and deoxypyridinoline (D-Pyr). To further assess bone metabolism during peripuerperal periods, we measured the urinary excretions of C-telopeptide (CTX) and cross-linked Ntelopeptide (NTX) of type I collagen, new markers of bone resorption. In addition to Pyr and D-Pyr, urinary CTX and NTX were measured by two ELISAs which recognize the corresponding peptide of type I collagen after urine samples were collected cross-sectionally from 230 women who consisted of 187 pregnants, 25 puerperants, and 18 age-matched nonpregnant women. Urinary CTX was also measured longitudinally from 10 pregnants at 5-9, 28-31 and 36-39 weeks of gestation and 1, 3, 6 months after parturition. Similar to the changes in Pyr and D-Pyr, the mean CTX and NTX values significantily increased in the 3rd trimester of pregnancy and remained high during puerperium compared with nonpregnant or early pregnant women $(P<0.05)$. In a longitudinal study, the mean CTX value significantly increased in the 3rd trimester of pregnancy and at 1 month of puerperium compared with that in the early stage of pregnancy $(P<0.05)$. These results further confirm our previous evidence that bone resorption is enhanced during the 3 rd trimester of gestation and puerperium and suggest that urinary CTX and NTX measured by ELISA, which is more convenient than HPLC, are useful markers to assess bone resorption during peripuerperal periods.
\end{abstract}

Key words: C-telopeptide (CTX), N-telopeptide (NTX), Bone resorption, Pregnancy, Puerperium

(Endocrine Journal 44: 733-738, 1997)

BY measuring urinary pyridinoline (Pyr), deoxypyridinoline (D-Pyr) and other biochemical markers of bone metabolism, we previously reported an increase in bone resorption accompanied by bone loss during pregnancy and lactation in healthy women [1]. Pyr and D-Pyr, known to be bone resorption markers, are crosslinks between mature collagen molecules, being primarily found in bone and cartilage but not in

\section{Received: March 24, 1997}

Accepted: July 4, 1997

Correspondence to: Dr. Michiyoshi TAGA, Department of Obstetrics and Gynecology, Yokohama City University School of Medicine, 3-9 Fukuura, Kanazawa-ku, Yokohama 236, Japan skin. D-Pyr is more specific for bone resorption than Pyr, but neither is absolutely bone-specific. The determination of the total excretion of Pyr and D-Pyr, however, can be used as an index of bone resorption. Several studies have shown that Pyr and D-Pyr are sensitive markers of bone resorption in postmenopausal women $[2,3]$ and in various metabolic bone diseases [4-6] characterized by increased bone turnover. Currently, Pyr and DPyr are measured fluorometrically in hydrolyzed urine after separation by high performance liquid chromatography (HPLC) [7], which is a timeconsuming and complicated method unsuitable for routine use. A monoclonal antibody recognizing free D-Pyr and exhibiting less than $2.5 \%$ crossreactivity with free Pyr has been developed and 
an enzyme-linked immunosorbent assay (ELISA) specific for urinary free D-Pyr is now available [8]. As a substantial fraction of Pyr cross-linking residues is excreted in peptide-bound form, others have recently developed immunoassays for Pyr cross-linked peptides [9].

Recently, bone resorption has been assessed by new markers for collagen degradation, Ctelopeptide (CTX) and cross-linked $\mathrm{N}$-telopeptide (NTX) of type I collagen. Bonde et al. described a new commercial ELISA that measures CTX, which has type I collagen-specific peptide sequences independent of the Pyr and D-Pyr structures [10]. Because $>90 \%$ of the organic matrix of bone consists of type I collagen, the peptide sequence measured in this assay is a potential marker of bone resorption. This marker is reported to reflect bone resorption and to be more sensitive than Pyr or DPyr as a marker of bone turnover [11]. The urinary level of NTX also has been reported to be a sensitive and specific marker of bone resorption [9, 12]. The few CTX and NTX data that have been reported so far are related to postmenopausal women [10, 11], metabolic bone diseases [11] and antiresorptive therapy [13]. There has been no report of measurements of urinary excretion of CTX and NTX during the peripuerperal period during which bone metabolism might undergo dynamic change. In both cross-sectional and longitudinal studies in part, we evaluated bone resorption during pregnancy and puerperium by measuring CTX and NTX with new ELISAs and compared findings with those for urinary Pyr and D-Pyr.

\section{Materials and Methods}

\section{Subjects and urine samples}

In the cross-sectional study, 230 women, 19 to 38 years of age $(28.8 \pm 3.5$, mean $\pm S D)$, consisting of 187 pregnant women (19-38 years of age, $28.9 \pm$ 3.4 , mean $\pm S D$ ), 25 puerperants (23-38 years of age, $30.5 \pm 4.0$, mean $\pm \mathrm{SD}$ ) and 18 nonpregnant women (22-30 years of age, $26.2 \pm 2.5$, mean $\pm S D$ ), were studied. Ten healthy pregnant women, 24 to 34 years of age $(28.1 \pm 2.7$, mean $\pm S D)$, completed the longitudinal and prospective study. For lactation, 6 women breast-fed for more than 3 months, whereas 4 women employed formula- feeding or breast-feeding for less than 3 months. None had a history of thyroid disease, renal disease, fracture or any diseases known to affect bone metabolism. Women in both the crosssectional and longitudinal studies had normal term deliveries and experienced no complications during pregnancy and puerperium, including toxemia, intra-uterine growth retardation, threatened abortion, threatened preterm labor or multiplet. Informed consent for participation in this study was obtained from all subjects.

One spot urine samples were collected in the morning in the cross-sectional study from 187 pregnant women throughout pregnancy and from 25 puerperants at 1 month postpartum as well as from 18 age-matched nonpregnant women. In the longitudinal study, urine samples were also collected in the morning from 10 patients first at 5-9 weeks gestation, then at 28-31 and 36-39 weeks gestation and at 1,3, and 6 months after parturition. The gestational week was confirmed by transvaginal ultrasonographic measurement of fetal crown-rump length in the early stage of pregnancy. Urine samples were frozen and kept at $-20{ }^{\circ} \mathrm{C}$ until they were assayed. All samples were assayed at one time at the end of the study to avoid interassay variability.

\section{Assay for CTX, NTX and other markers in urine}

Urinary excretion of CTX was measured by ELISA with CrossLapsTM ELISA (Osteometer A/S, R $\phi$ ovre, Denmark) by slightly modifying Garnero's method [11]. Anti-CTX antibody was obtained by immunizing rabbits with an amino acid sequence specific for part of the C-terminal telopeptide of the $\alpha 1$ chain of type I collagen (Glu-Lys-Ala-HisAsp-Gly-Gly-Arg) (8AA) conjugated to bovine serum albumin by a two-step carbodimide procedure. The concentration of 8AA equivalents in each subject's specimen was determined by interpolation on the calibration curve. The intraassay and interassay CVs were $<13 \%$ in the concentration range of the calibration curve [11]. The data were corrected for creatinine $(\mathrm{Cr})$ excretion determined enzymatically with picric acid and expressed as $\mu \mathrm{g} / \mathrm{mmol} \mathrm{Cr}$.

Urinary NTX was quantified directly in unextracted urine by ELISA with a specific monoclonal antibody to NTX [9]. The ELISA kit 
was produced by Mochida Pharmaceutical Co. Ltd. (Tokyo, Japan) according to the modified method of Eyre and supplied for clinical trial use. Assay values are standardized to an equivalent amount of bone collagen, and are expressed in nanomolar bone collagen equivalent per millimolar $\mathrm{Cr}(\mathrm{nmol}$ $\mathrm{BCE} / \mathrm{mmol} \mathrm{Cr}$ ). Sensitivity of the assay is $20 \mathrm{nmol} /$ $l \mathrm{BCE}$. Intra- and interassay variability averages were $4.6 \%$ and $4.1 \% \mathrm{CV}$, respectively.

The total urinary excretions of Pyr and D-Pyr were measured by HPLC as previously described [7] and the data were corrected for $\mathrm{Cr}$ and expressed as $\mathrm{nmol} / \mathrm{mmol} \mathrm{Cr}$.

Duplicate measurements were performed for each urine sample.

\section{Statistical analysis}

The statistical significance of the differences between mean group values at a number of time points was determined by repeated measures analysis of variance (ANOVA) followed by numerous comparison tests. Dunnett's test was used for comparison of mean values in the crosssectional study and Dunnett-Hsu's test was used in the longitudinal study. Calculations were performed with the SAS statistical programs (SAS Institute; Carey, NC).

\section{Results}

\section{Changes in urinary CTX and NTX during pregnancy and puerperium in the cross-sectional study}

Cross-sectional changes in the mean ( \pm SEM) CTX, NTX, Pyr and D-Pyr values in nonpregnant women, women during pregnancy and at 1 month postpartum are illustrated in Fig. 1, Fig. 2, Fig. 3 and Fig. 4, respectively. The pregnant women were divided into 9 groups according to the week of gestation, with 14-29 women in each group. The profiles of the changes were similar for all of these four markers. Pyr and D-Pyr significantly increased after the 3rd trimester of pregnancy and at 1 month postpartum compared with the values for nonpregnant women or women in the early stages of pregnancy $(P<0.05)$. CTX and NTX levels also increased during similar periods, although a statistically significant increase was observed only at 32-35 weeks of gestation in CTX (Fig. 1).

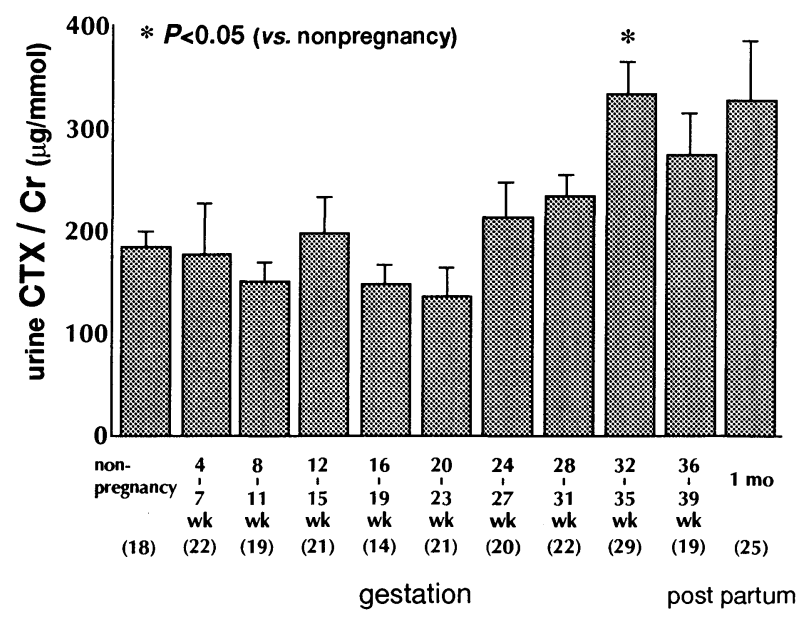

Fig. 1. Cross-sectional change in the mean ( \pm SEM) levels of urinary CTX measured by ELISA during pregnancy and puerperium in 18 nonpregnant women, 187 pregnant women, and 25 puerperants. The pregnant women were divided into 9 groups according to the week of gestation, with 14-29 women in each group. The mean CTX value increased more than 20\% after 28-31 weeks of pregnancy compared to that in nonpregnant women, although a significant increase was observed only at $32-35$ weeks $(P<0.05)$.

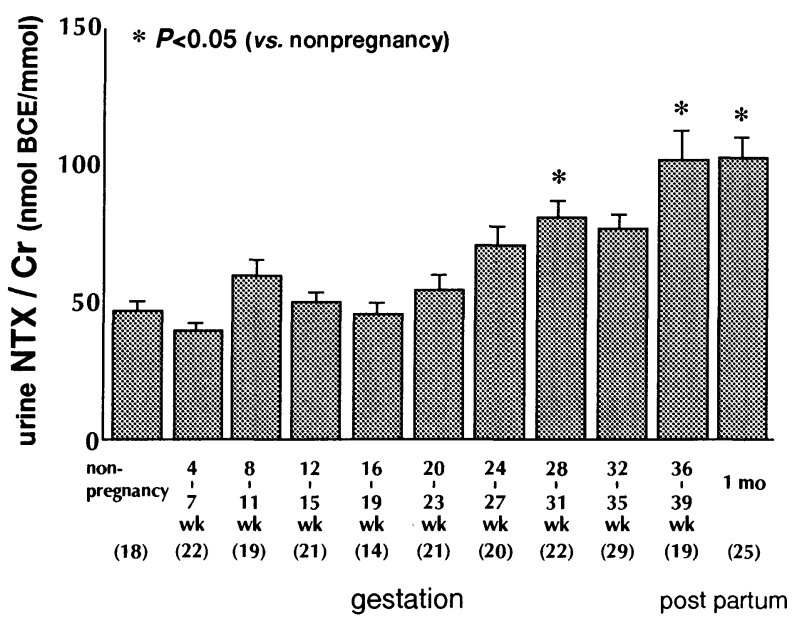

Fig. 2. The mean levels of urinary NTX during pregnancy and puerperium. The mean NTX level significantly increased at 28-31, 36-39 weeks of pregnancy and 1 month postpartum compared with that in nonpregnant women $(P<0.05)$. 


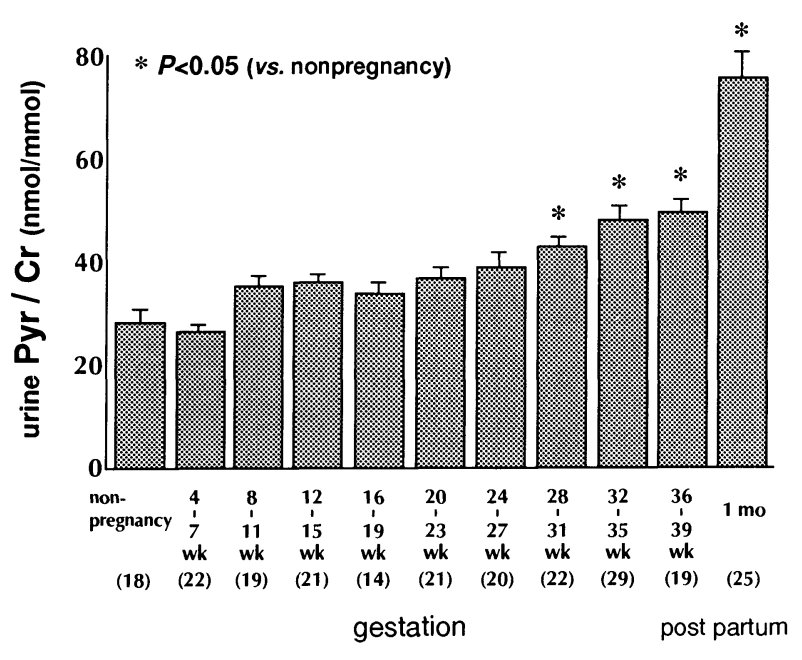

Fig. 3. Urinary excretions of pyridinoline (Pyr) during pregnancy and puerperium. The excretion of urinary Pyr was significantly increased at 28-39 weeks of gestation and 1 month postpartum compared with that in nonpregnant women $(P<0.05)$.

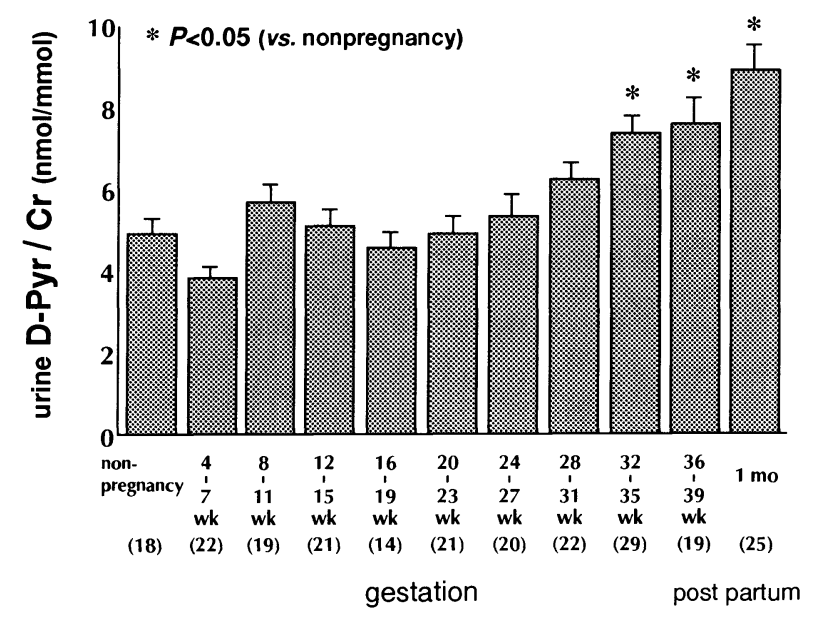

Fig. 4. The mean urinary deoxypyridinoline (D-Pyr) during pregnancy and puerperium. Compared with that in nonpregnant women, the urinary D-Pyr level was significantly increased at 32-39 weeks of pregnancy and 1 month postpartum $(P<0.05)$.

\section{Change in urinary CTX during pregnancy and puerperium in the longitudinal study}

Figure 5 shows the longitudinal change in the mean ( \pm SEM) urinary CTX values throughout pregnancy and puerperium up to 6 months in 10 pregnant women. The mean CTX value significantly increased at 28-31 and 36-39 weeks of pregnancy and at 1 month of puerperium

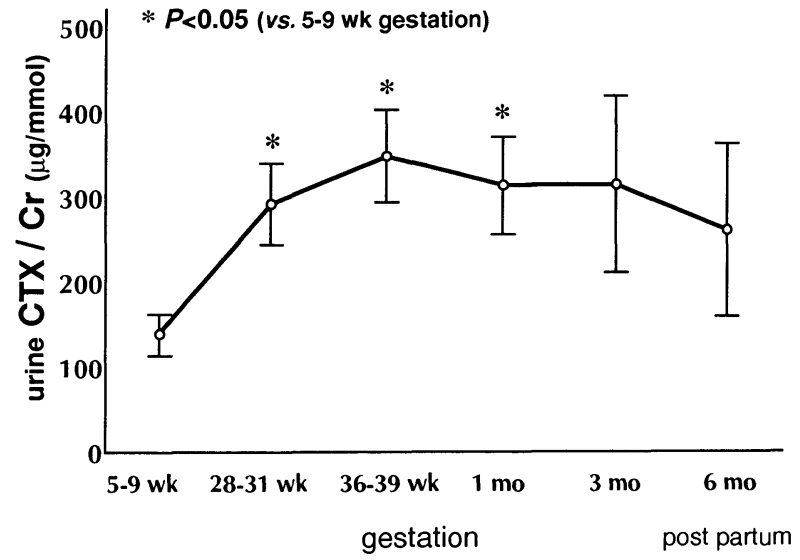

Fig. 5. Longitudinal changes in mean ( \pm SEM) levels of urinary excretion of CTX measured by ELISA throughout pregnancy and puerperium in 10 healthy women. The mean CTX value was significantly increased at 28-31 and 36-39 weeks of gestation compared with that at $5-9$ weeks $(P<0.05)$ and remained at a high level during puerperium.

compared with that in the early stage of pregnancy $(P<0.05)$. Although the CTX level was still high during 3-6 months of puerperium, the difference was not statistically significant compared with the early stage of pregnancy.

\section{Discussion}

We previously showed an acceleration in bone metabolism during pregnancy and lactation because not only serum levels of alkaline phosphatase and intact-osteocalcin but also levels of urinary excretion of Pyr and D-Pyr, markers of bone resorption, were increased after 28 weeks of gestation and during puerperium [1]. Urinary Pyr and D-Pyr are good parameters of bone resorption, but their measurement by HPLC is timeconsuming, complicated and unsuitable for routine use. Recently new bone resorption markers such as CTX [10] and NTX [9] have been developed. CTX ELISA performs well with respect to precision, recovery and dilution of urine samples, providing a convenient and useful index of bone resorption, such as detection of the increase in bone turnover associated with menopause [11]. Hanson et al. [9] reported that the assay method for NTX was considerably promising as a rapid and specific noninvasive index of human bone resorption rates. NTX assay also provided greatly improved 
specificity and convenience over total pyridinoline analysis [9]. In CTX, the major reason for selecting this part of the molecule is that the sequence contains an important region for intermolecular cross-links and that the proximity of cross-linking residues and the compact structure of these molecules may protect such peptides from further renal degradation [10].

In this study, we analyzed bone resorption during pregnancy and puerperium by evaluating urinary biochemical parameters of bone metabolism, including the new markers CTX and NTX, in both cross-sectional and longitudinal methods. With these ELISAs in a cross-sectional study, we have shown for the first time that urinary CTX and NTX are significantly higher in the 3rd trimester of pregnancy than those in nonpregnancy or the early stage of pregnancy and remain high during puerperium. These gestational and puerperal changes in urinary CTX and NTX excretions were similar to those in Pyr and D-Pyr. Although CTX and NTX have been reported to be more sensitive than Pyr or D-Pyr [9-12], this is not necessarily in agreement with our results. One reason may be that we employed a cross-sectional study, in which data vary more than in a longitudinal study. Another reason seems to be that CTX and NTX values in pregnant or puerperal women vary more than in postmenopausal and osteoporotic women, for whom most CTX and NTX were measured previously. The CTX value at 2831 and 36-39 weeks of gestation and at 1 month postpartum was also higher than that in 5-9 weeks of gestation in our longitudinal study. The longitudinal profile of CTX during pregnancy and puerperium is similar to those of Pyr and D-Pyr, which were shown by our previous study [1]. These data suggest that bone resorption is enhanced during the 3rd trimester of pregnancy and puerperium, confirming our previous results obtained from the longitudinal analysis of Pyr, DPyr and hydroxyproline [1]. Although similar profiles of CTX excretion during pregnancy and puerperium were obtained from longitudinal and cross-sectional studies, there was a small difference between these two studies. A longitudinal study will provide more reliable results because a crosssectional study will not adequately reveal small changes in CTX which may vary greatly from subject to subject. Because urinary CTX and NTX can be measured by ELISA, which is more convenient than HPLC, these two new markers for bone resorption are useful for assessing bone resorption during peripuerperal periods.

Our confirmation of enhancement of bone resorption during pregnancy and puerperium by the new ELISAs for CTX and NTX suggests the possibility of loss of bone mineral density during these periods. Several investigators, including ourselves, have reported that bone mineral density decreases during pregnancy or puerperium $[1,14-$ 19]. As we mentioned previously [1], the reason for increased bone turnover during pregnancy despite a hyperestrogenic environment is unclear. One possibility is that the serum 1,25-dihydroxy vitamin $\mathrm{D}$ level is greatly increased during pregnancy $[20,21]$ with serum parathyroid hormone or calcitonin being unchanged [22, 23]. The mechanism of high bone turnover during puerperium is also uncertain, but it may be related to breast-feeding which is accompanied by a hyperprolactinemic and hypoestrogenic state [24, 25]. We have previously shown that urinary Pyr and D-Pyr in breast-feeding women were significantly higher than those in formula-feeding women [1]. Kent et al. reported that plasma alkaline phosphatase, serum osteocalcin and urine hydroxyproline were significantly higher in lactating women than in controls [17].

In conclusion, we have shown that the urinary CTX and NTX levels, like Pyr and D-Pyr, increase after 28 weeks of gestation and remain high until 1 month postpartum. This new evidence confirms the results of our previous study showing an enhancement of bone resorption during the latter part of gestation and puerperium and also indicates that urinary CTX and NTX determined by ELISA are useful markers during the peripuerperal periods because of convenience in measuring.

\section{References}

1. Yamaga A, Taga M, Minaguchi H, Sato K (1996) Changes in bone mass as determined by ultrasound and biochemical markers of bone turnover during pregnancy and puerperium: A longitudinal study. 
J Clin Endocrinol Metab 81: 752-756.

2. Uebelhart D, Schlemmer A, Johansen JS, Gineyts E, Christiansen C, Delmas PD (1991) Effect of menopause and hormone replacement therapy on the urinary excretion of pyridinium cross-links. J Clin Endocrinol Metab 72: 367-373.

3. Schlemmer A, Hassager C, Jensen SB, Christiansen C (1992) Marked diurnal variation in urinary excretion of pyridinium cross-links in premenopausal women. J Clin Endocrinol Metab 74: 476-480.

4. Uebelhart D, Gineyts E, Chapuy MC, Delmas PD (1990) Urinary excretion of pyridinium crosslinks: A new marker of bone resorption in metabolic bone disease. Bone Miner 8: 87-96.

5. Delmas PD, Gineyts E, Bertholin A, Garnero P, Marchand F (1993) Immunoassay of pyridinoline crosslink excretion in normal adults and in Paget's disease. J Bone Miner Res 8: 643-648.

6. Garnero P, Vassy V, Bertholin A, Riou JP, Delmas PD (1988) Markers of bone turnover in hyperthyroidism and the effects of treatment. J Clin Endocrinol Metab 78: 955-959.

7. Black D, Duncan A, Robins SP (1988) Quantitative analysis of the pyridinium crosslinks of collagen in urine using ion-paired reversed-phase highperformance liquid chromatography. Anal Biochem 169: 197-203.

8. Daniloff GY, Hesley RP, Ju J, Evans BJ, He P, Seyedin SM (1993) An immunoassay for deoxypyridinoline. A highly specific marker of bone resorption. J Bone Miner Res 8: S357.

9. Hanson DA, Weis MA, Bollen AM, Maslan SL, Singer FR, Eyre DR (1992) A specific immunoassay for monitoring human bone resorption: Quantitation of type I collagen cross-linked N-telopeptides in urine. J Bone Miner Res 7: 1251-1258.

10. Bonde M, Qvist $P$, Fledellius $C$, Riis BJ, Christiansen C (1994) Immunoassay for quantifying type I collagen degradation products in urine evaluated. Clin Chem 40: 2022-2025.

11. Garnero P, Gineyts E, Riou JP, Delmas PD (1994) Assessment of bone resorption with a new marker of collagen degradation in patients with metabolic bone disease. J Clin Endocrinol Metab 79: 780-785.

12. Gertz BJ, Shao P, Hanson DA, Quan H, Harris ST, Genant HK, Chesnut III CH, Eyre DR (1994) Monitoring bone resorption in early postmenopausal women by an immunoassay for cross-linked collagen peptide in urine. J Bone Miner Res 9: 135-142.

13. Garnero P, Gineyts E, Arbault P, Christiansen C, Delmas PD (1995) Different effects of biophosphonate and estrogen therapy on free and peptide-bound bone cross-links excretion. J Bone
Miner Res 10: 641-649.

14. Akinson PJ, West RR (1970) Loss of skeletal calcium in lactating women. J Obstet Gynaecol Br Commonw 77: $555-560$.

15. Lamke B, Brundin J, Moberg P (1977) Changes of bone mineral content during pregnancy and lactation. Acta Obstet Gynecol Scand 56: 217-219.

16. Hayslip CC, Klein TA, Wray HL, Duncan WE (1989) The effects of lactation on bone mineral content in healthy postpartum women. Obstet Gynecol 73: 588592.

17. Kent GN, Price RI, Gutterrige DH, Smith M, Allen JR, Bhagat CI, Barlnes MP Hickling CJ, Retallack RW, Wilson SG, Devlin RD, Davies C, Jhon AS (1990) Human lactation: Forearm trabecular bone loss, increased bone turnover, and renal conservation of calcium and inorganic phoshate with recovery of bone mass following weaning. $J$ Bone Miner Res 5: 361-369.

18. Drinkwater BL, Charles H (1991) Bone density changes during pregnancy and lactation in active women: A longitudinal study. Bone Miner 14: 153160.

19. Sowers MF, Corton G, Shapiro B, Jannarsch ML, Crutchfield M, Smith ML, Randolph JF, Hollis B (1993) Changes in bone density with lactation. JAMA 269: 3130-3135.

20. Reddy GS, Norman AW, Willis DM, Goltzman D, Guyda H, Solomon S, Philips DR, Bishop JE, Mayer E (1983) Regulation of vitamin D metabolism in normal human pregnancy. J Clin Endocrinol Metab 56: 363-370.

21. Wilson SG, Rtallack RW, Kent JC, Worth GK, Gutterridge DH (1990) Serum free 1,25-dihyroxyvitamin $\mathrm{D}$ and the free 1,25-dihydroxyvitamin $\mathrm{D}$ index during a longitudinal study of human pregnancy and lactation. Clin Endocrinol 32: 613622.

22. Davis OK, Hawkins DS, Rubin LP, Posillico JT, Brown EM, Schiff I (1988) Serum parathyroid hormone (PTH) in pregnant women determined by an immunoradiometric assay for intact PTH. J Clin Endocrinol Metab 67: 850-852.

23. Seki K, Makimura N, Mitsui C, Hirata J, Nagata I (1991) Calcium-regulating hormones and osteocalcin levels during pregnancy: A longitudinal study. Am J Obstet Gynecol 164: 1248-1252.

24. Battin DA, Marrs RP, Fleiss PM, Mishell DR (1985) Effect of suckling on serum prolactin, luteinizing hormone, follicle-stimulating hormone, and estradiol during prolonged lactation. Obstet Gynecol 65: 785-788.

25. Dada OA, Laditan AAO (1982) Circulating hormonal levels during prolonged lactational amenorrhea. Clinica Chimica Acta 123: 287-292. 